\title{
SWARM: A regional health system's intervention approach to COVID-19 outbreaks in nursing and adult care homes in rural eastern North Carolina
}

\author{
Gregory D. Kearney*1, Pamela Cowin ${ }^{2}$, Todd Hickey², Bennett Wall ${ }^{2}$, Jeffrey Shovelin ${ }^{2}$, Michael Waldrum ${ }^{1,2}$, Debra \\ Thompson ${ }^{2}$ \\ ${ }^{1}$ East Carolina University, Brody School of Medicine, United States \\ ${ }^{2}$ Vidant Health, Greenville, NC, United States
}

Received: July 17, 2020

DOI: $10.5430 /$ jha.v9n4p27
Accepted: August 10, 2020

Online Published: August 24, 2020

\begin{abstract}
Background: Incessant, COVID-19 outbreaks occurring in nursing and adult care homes are a serious public health concern that continues to create significant healthcare crisis management challenges. Adult care facilities often lack in-house capacity and capability to safely treat its ill residents, while hospitals are strained to balance the influx of patients, allocate scarce resources and protect healthcare workers.

Objectives: This project sought to implement a regional, community engaged, intervention model to assist nursing and adult care homes in reducing or preventing outbreaks and risks associated with COVID-19 in rural eastern North Carolina (N.C.).

Methods: Design/Setting: Through collaborations between Vidant Health (VH), health departments and a network of community partners, a shared intervention plan was created and implemented to monitor nursing and adult care homes for COVID-19-related outbreaks across 29 counties in rural eastern N.C. A "Strike" team or "Swarm (SWARM) approach was developed as an operationalized concept for rapidly responding to nursing and adult care home outbreaks while providing an array of services and interventions to help prevent the spread of COVID-19. Comparative analysis was conducted between the mean number of COVID-19-related cases, deaths and length of outbreak time in VH service contracted, SWARM facilities $(\mathrm{n}=12)$ and all other non-service contracted, or non-SWARM facilities $(n=155)$ in N.C.

Results: Nursing and adult care homes under service contract using our SWARM approach experienced fewer average number of COVID-19-related resident ill cases (24.4 vs 29.0), and deaths (1.2 vs. 3.9). The length of outbreak recovery time was far less among SWARM facilities than non-participating, non-SWARM facilities $(17.1$ vs. $25.4 ; p<.034)$.

Conclusions: By actively monitoring key indicators, engaging in daily communication with local partners and providing rapid response, VH's SWARM approach provides a proactive method for preventing further spread of COVID-19 in adult care facilities and communities.
\end{abstract}

Key Words: Administration, Management, North Carolina, Population health

\footnotetext{
*Correspondence: Gregory D. Kearney; Email: KearneyG@ ecu.edu; Address: East Carolina University, Brody School of Medicine, Dept. of Public Health, 115 Heart Drive, MS 660 Greenville, NC 27834, United States.
} 


\section{INTRODUCTION}

In February 2020, the Life Care Center in Kirkland, Washington (King County) became the epicenter of coronavirus disease (COVID-19) in the U.S. The outbreak gained national attention resulting in over 100 associated positive cases, and upwards of 30 deaths. ${ }^{[1]}$ Findings by local and federal health officials later identified the spread of the virus primarily as the result of multiple factors, including ineffective infection control and prevention, failure to notify the department of health of the outbreak in a timely manner; staff members working in multiple facilities, and lack of back-up clinicians for the facility. ${ }^{[1]}$

Shortly following the King County incident, the number of outbreaks and deaths related to COVID-19 in adult care facilities across the U.S. skyrocketed, sending an alarming outpouring of residents and staff to hospitals. ${ }^{[2]}$ In response, hospitals all over the country ramped up efforts to respond to the pandemic surge, cancelling elective and non-essential surgeries to free up medical workers, seeking scarce personal protective equipment (PPE), ventilators, and additional bed space. ${ }^{[3]}$ Many hospitals and healthcare centers encountered "apocalyptic" conditions and were besieged by hundreds of COVID-19 patients, many of whom were elderly and later died. ${ }^{[4]}$

Ill-patient transfer from nursing homes to medical centers presents multiple hazards for both the treating hospital and the adult care facility. ${ }^{[5]}$ The influx of ill-patients into hospitals and emergency departments can strain resources, overburden healthcare workers and increase risk of intra-hospital patient transmission, or nosocomial spread to other patients. ${ }^{[6]}$ Nursing homes face their own dilemma, often lacking capacity and capability to safely monitor older patients as they transition from the hospital back to the facility. ${ }^{[7]}$ Resolving ill-patient transfer prior to pandemic conditions requires necessary and prudent decision making that requires thoughtful planning, coordination and communication by both parties. $^{[8,9]}$

In May 2020, news media reports estimated that over $61 \%$ of COVID-19-related deaths in N.C. were attributed to outbreaks in adult care facilities, ${ }^{[10]}$ citing multiple health code violations, including improper infection control procedures as part of the investigations. ${ }^{[11]}$ With over 400 nursing homes, 36,000 residents and 25,000 employees in adult care facilities in N.C. ${ }^{[12]}$ the threat of COVID-19 is a recognizable and imminent concern for hospitals and healthcare centers that treat older adults, ${ }^{[13]}$ as well as nursing home administrators, staff, families and communities. Recognizing these concerns, administrators at Vidant Health (VH), a regional healthcare system in rural eastern N.C., worked to establish partnerships with key community stakeholders, including local health departments and nursing home administrators to address this issue. Over the duration of several weeks and many workgroup meetings, an integrated, community COVID-19 monitoring and action response plan, referred to as "Swarm", or SWARM, was launched. Similar to a "strike team," the objectives of SWARM are to monitor and rapidly assist facilities with the goals of preventing outbreaks, containing spread, and reducing risk through a "treatment-inplace" model and the prevention of unnecessary transfers of COVID-19 positive patients to either the emergency department and/or acute care hospital.

\section{Project aims}

The aims of this project were to evaluate our SWARM approach by comparing differences between reported COVID19-related outbreak recovery periods, cases and deaths in nursing and adult care homes in N.C. that maintained service contracts using the SWARM approach, with other facilities in N.C. that did not use the SWARM approach.

\section{METHODS}

\subsection{Vidant Health}

Vidant Health $(\mathrm{VH})$ is a not-for-profit, regional healthcare system that encompasses a level 1 trauma center and eight (8) community hospitals, more than 14,000 employees and serves more than 1.4 million people across 29 rural counties in eastern N.C. Vidant Medical Center (VMC), located in Greenville N.C., is the heart of the Vidant Health system and serves as the medical teaching hospital for East Carolina University, Brody School of Medicine.

\subsection{Framework}

Through a combination of experience, evidence-based literature and discussions between $\mathrm{VH}$ administrators and stakeholders, the creation of the COVID-19 Care Pathway (CCP) was developed as the operational framework for this project (see Figure 1). The foundational elements of the CCP are to ensure that timely, safe and reliable access to resident treatment is prioritized, while emphasizing a "treatment-in-place" plan approach to avoid unnecessary hospital visits among senior living facilities (SNFs) and assisted living facilities (ALFs) residents. Throughout the CCP development process, strong emphasis was placed on patient safety and quality of care, communication and prevention. The CCP served, and continues to serve as a collaborative, operational reference guide to stakeholders, including county health departments, care partners, and community organizations to prevent the spread of COVID-19 in eastern N.C. 


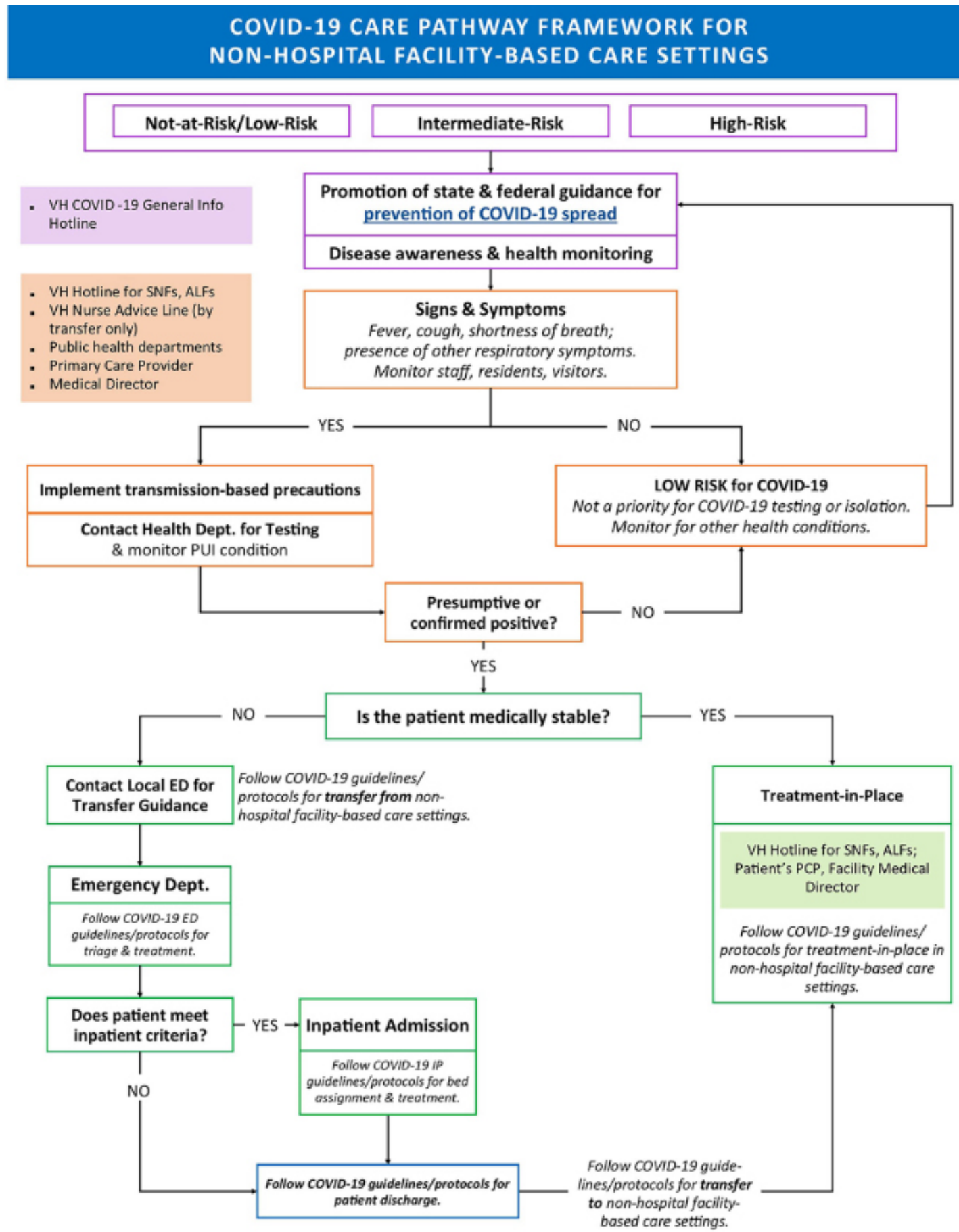

Figure 1. Care Pathway Framework

\subsection{SWARM}

A major component of the CCP is VH's SWARM and SWARM teams. As an operationalized concept, SWARM provides rapid response and an array of services and interventions to help prevent the spread of COVID-19 in nursing and adult care homes. Assessment and risk mitigation strategies of the SWARM approach are purposefully kept transparent and shared across stakeholders including VH's regional Published by Sciedu Press hospitals, facility administration, facility medical director, local health department leadership, and where appropriate, community stakeholders.

\subsection{Surveillance and response}

One of the key components of SWARM's team monitoring and response planning is early execution of a service contract agreement with nursing and adult care facilities prior to a trig- 
gering event. As further described below, having a contract with a facility allows VH to provide an array of services such as being able to cover shifts due to staffing shortages, provide PPE, conduct resident/staff testing, monitor and respond to a COVID-19 outbreak. An executed service agreement also ensures VH's SWARM teams can work closely with the local facility to assess the greatest risks and needed areas of support. Active surveillance of facilities are conducted daily through communication between VH's Transitional Care Coordinators, the local facility administrator and/or Director of Nursing, the local health department, and the local medical director for the facility. Daily, routine facility check-ins by Transitional Care Coordinator's and regional SWARM leadership with facilitators includes a review of the following: lab test results, monitoring changes to community risk level such as the number of cases in the community, evaluating the nurse triage call volume activity, surveying for increases/decreases in signs and symptoms among staff and residents, identifying staff on leave due to illness or potential exposure, determining the number of patients on isolation at the facility due to COVID-19 signs/symptoms, evaluating infection and control risk, such as PPE availability and shortages, identification of staff members working at multiple facilities, and determining any outbreaks at nearby facilities.

\subsection{Data}

To gauge the impact of our SWARM interventions, a comparative analysis was conducted among all N.C., nursing and adult care homes that had a N.C. Department of Health and Human Services (NC DHHS) reportable COVID-19-related outbreak between May 1 to June 12, 2020.

Publicly available data for the analysis was obtained from bi-weekly reports on the NC DHHS website. ${ }^{[14]}$

\subsection{Procedures and measures}

Reported outbreak data for NC nursing and adult care homes were grouped into two (2) categories; SWARM and NonSWARM facilities. SWARM facilities were those that maintained an executed service agreement or were monitored daily by VH; Non-SWARM facilities were all other remaining NC facilities that reported an outbreak but did not maintain a contract agreement with or have routine monitoring in place by $\mathrm{VH}$.

Selected outcome measures included the number of nursing homes and residential care facilities with a reported outbreak among the following: 1) number of days the outbreak lasted (until recovery, or no longer on the report), 2) number of cases among residents and staff, and the 3) number of deaths among residents and staff. The recovery time for an outbreak was calculated by summing the days between reporting periods for each facility This project was reviewed by East Carolina University Institutional Review Board.

\subsection{Analysis}

Univariate and non-parametric tests were used in data analysis. The Mann-Whitney U test was used to compare differences between the length of time (number of days) an outbreak occurred. Statistical significance was set at the 0.05 alpha level. Data analysis was conducted using SPSS v24.

Table 1. Number of reported COVID-19 cases, deaths and days until recovery among SWARM (service contracted facility) and non-SWARM (non-service contracted facility) nursing and adult care homes ( $=167$ ), North Carolina, 2020

\begin{tabular}{|c|c|c|c|c|c|c|c|}
\hline \multirow{3}{*}{ Facilities Reporting an Outbreak } & \multicolumn{7}{|c|}{ Type of Group Facility } \\
\hline & \multicolumn{3}{|c|}{$\begin{array}{c}\text { SWARM n (\%) } \\
12(7.2)\end{array}$} & \multicolumn{3}{|c|}{$\begin{array}{c}\text { Non-SWARM n (\%) } \\
155(92.8)\end{array}$} & \multirow[t]{2}{*}{$p$} \\
\hline & $n$ & $M$ & $S D$ & $n$ & $M$ & $S D$ & \\
\hline Cases (total) & 293 & 24.4 & 28.4 & 4527 & 29.0 & 35.4 & .762 \\
\hline Residents & 200 & 16.7 & 20.0 & 3148 & 20.2 & 26.0 & .904 \\
\hline Staff & 93 & 7.8 & 8.5 & 1379 & 8.8 & 10.6 & .953 \\
\hline Deaths (total) & 14 & 1.2 & 2.2 & 624 & 4.0 & 6.6 & .762 \\
\hline Residents & 14 & 1.2 & 2.2 & 621 & 3.9 & 6.6 & .308 \\
\hline Staff & NR & NR & NR & 3 & 0.0 & 0.1 & .953 \\
\hline Number of Days Until Recovery & 205 & 17.1 & 14.3 & 3905 & 25.4 & 13.4 & .032 \\
\hline
\end{tabular}

Note. $\mathrm{NR}=$ None Reported. Cases and deaths are the maximum number reported. Recovery for these study purposes followed NC DHHS, rules and guidelines as follows; a) a COVID-19 outbreak is defined as two or more laboratory-confirmed cases; b) An outbreak is considered when there no evidence of continued transmission within the facility, measured as 28 days after the latest date of onset in a symptomatic person or the latest date of a specimen collection in an asymptomatic person, whichever is later, and c) Facilities with less than 10 residents are not included to protect patient confidentiality. 


\section{RESUltS}

As shown in Table 1, there were a total of 167 nursing and adult care facilities reporting an outbreak in N.C. during the specified time period. Among the total number of facilities reporting an outbreak, $7.2 \%(\mathrm{n}=12)$ were SWARM service contracted facilities and $92.8 \%(\mathrm{n}=155)$ non-SWARM, non-service contracted facilities.

The average number of reported COVID-19 resident cases among SWARM facilities was 16.7, compared to 20.2 residents cases in non-SWARM facilities. Among staff member COVID-19 cases, SWARM had an average of 7.8 versus 8.8 staff member cases in non-SWARM facilities. The average number of deaths among residents was 1.2 in SWARM facilities compared to 4.0 residents in non-SWARM facilities. There were no reported staff member deaths in SWARM facilities, and 3.0 staff member deaths in non-SWARM facilities.

The average number of days until recovery of an outbreak in SWARM facilities was 17.1 days compared to 25.4 days in non-SWARM facilities. A Mann-Whitney U test indicated that this difference was statistically significant $(p<.034)$.

\section{Discussion}

These findings suggest that our SWARM approach for actively monitoring key factors in nursing and adult care homes contributed to fewer COVID-19 illnesses, deaths and an overall quicker recovery time from outbreaks than those that were not aligned with SWARM.

Our collaborative efforts to monitor and respond to COVID19 outbreaks in nursing and adult care homes on a regional scale facilitated by a not-for-profit health system may be the first in the U.S. These results are encouraging and build on evidence-based approaches, for treating patients on-site successfully as demonstrated with other health conditions, for example, pneumonia. ${ }^{[5]}$ Efforts to combat the rapid threat of COVID-19 in congregate style living facilities have been increasingly evolving throughout the U.S. For example, Maryland and other states are expanding traditional state emergency management services (EMS) roles by partnering with local health departments and providers to lead "strike teams", ${ }^{[15]}$ similar to SWARM. These types of innovative and efficient efforts to curb COVID-19 outbreaks in adult congregate living facilities are excellent examples that should be recognized and further considered as national model programs

\subsection{Implications and future research}

While the results of this project highlight the need for rapid interventions to reduce COVID-19 in these facilities, there are exceedingly dynamic challenges that should be consid- ered when conducting future research on this topic. Monitoring for risk factors and prioritizing the needs of senior adult care facilities changes daily as the virus spreads across communities and the risk for vulnerability of high-risk patients rise and fall. Environmental factors play a significant role in viral transmission are also continually evolving. For example, personal hygiene such as handwashing, knowledge and use of PPE, effective infection and control measures vary by individual facility and are highly influenced by staff members education and personal behavior.

As demonstrated in the King County case, staff facility members working in multiple facilities, working while symptomatic and inadequate familiarity with PPE were major contributing factors leading up to the outbreak. ${ }^{[1]}$ Grabowski and colleagues described part of the challenge health administrators are confronted with is the ability to find high-quality staff employees that are willing to work in these settings, and further noted that this issue would be magnified in the context of COVID-19. ${ }^{[7]}$ Similar issues involving staff, particularly working in multiple facilities have been identified in outbreak follow up reports. While it remains vitally important for administrators to have staff tested regularly for symptoms to ensure they are not spreading the virus, this issue is sometimes met with cost and logistic challenges. In light of this issue, as the authors were finalizing this paper, N.C. lawmakers implemented mandatory testing for all nursing home residents and employees regardless of symptoms or whether there is an active outbreak at a facility. ${ }^{[12]}$

Given the complexity of virus transmission, it is difficult to determine with accuracy which part of SWARM's intervention methods had the most significant impact on reducing viral spread and shortening the length of outbreak recovery time. Nevertheless, our summation is that having an executed service agreement in place with facilities and daily focused monitoring using the SWARM approach correlated with fewer cases, deaths and shorter recovery time. As demonstrated, our experience shows that by having an agreement in place improves upon the ability to conduct daily monitoring, to treat ill-patients "in-place" and helps to "open the door" for communication with facility administrators. In light of the agreement with facilities, communication and supporting routine health inspection reports from local health departments helped to signal early warnings or outbreaks for rapid response.

The SWARM approach to protect facilities from COVID entry, containing spread, and "treating-in-place" are guided by over-arching state, federal policies and best-practice recommendations. At a more granular level, however, practicebased approaches and guidelines are needed to supplement these documents. More published case studies and case re- 
ports are needed to help reduce outbreaks, guide policy and action. Recognizing how quickly the science is developing on COVID-19 underscores the need to maintain current response plans, training, and education on a consistent and regular basis for administrators, healthcare workers and staff.

\subsection{Limitations}

Considering these findings, several factors should be recognized when interpreting results of our data analysis. It was unknown if other underlying health co-morbidities were the major cause of reported cases and deaths or were the direct result of COVID-19, which limits the accuracy of these results. The data provided did not include the total number of residents/staff members in facilities, limiting the ability to calculate and compare accurate rates. Additionally, the state's case/death reports did not include data on facilities with 10 or less residents. If this data should become available, subsequent analysis should include calculated rates, number of cases and deaths in smaller facilities and a longer study period.

\section{Conclusions}

With an estimated 1.3 million individuals residing in nursing homes and nearly one in 10 reporting cases of COVID-19 in the US, ${ }^{[16]}$ proactive measures are needed to reduce illnesses and deaths among older adults in these high-risk, congre- gate living environments. ${ }^{[17]}$ Hospitals collaborating with key partners, play a vital role in facilitating necessary action to bring change to protect this sensitive population group. Our SWARM approach is a shared model that can assist hospitals, healthcare and congregate living facilities increase stakeholder partnerships and protect communities from the spread of COVID-19.

\section{Implications for policy and practice}

- Hospitals and community partners working together can play a vital role in the prevention and spread of COVID-19 in congregate living facilities and communities.

- Active involvement by facility medical directors and administrators in staff screening and training, infection control measures, PPE utilization are important components leading up to and controlling an outbreak.

- Over-arching state and federal guidelines are important for administrators in preparing and responding to outbreaks in adult care facilities. Practice-based examples, such as case studies, and tabletop exercises are needed to train and educate staff and assist decision makers in addressing day-to-day operations.

\section{CONFlicts of InTEREST Disclosure}

The authors declare they have no conflicts of interest.

\section{REFERENCES}

[1] McMichael TM, Clark S, Pogosjans S, et al. COVID-19 in a longterm care facility - King County, Washington, February 27-arch 9, 2020. MMWR Morb Mortal Wkly Rep. 2020; 69(12): 339-342. PMid: 32214083. https://doi.org/10.15585/mmwr.mm6912 e1

[2] Cenziper D, Jacobs J, Mulcahy S. Nearly 1 in 10 nursing homes nationwide report coronavirus cases. Washington Post. Business section. 2020 [Accessed May 14, 2020]. Available from: https://www. washingtonpost. com/business/2020/04/20 /nearly-one-10-nursing-homes-nationwide-report-cor onavirus-outbreaks/

[3] Klein MJ, Frangos SG, Krowsoski L, et al. Acute care surgeons response to the COVID-19 pandemic: Observations and strategies from the epicenter of the American crisis. Ann Surg. 2020. https://doi.org/10.1097/SLA.0000000000004028

[4] Dwyer J. One hospital was besieged by the virus. Nearby was "plenty of space". New York Times. About New York section. May 142020 [Accessed May 19, 2020]. Available from: https://www.nytimes.com/2020/05/14/nyregion/co ronavirus-ny-hospitals.html

[5] Loeb M, Carusone SC, Goeree R, et al. Effect of a clinical pathway to reduce hospitalizations in nursing home residents with pneumonia: A randomized controlled trial. JAMA. 2006; 295(21): 2503-
2510. PMid: 16757722. https://doi.org/10.1001/jama. 295 .21 .2503

[6] Klompas M. Coronavirus disease 2019 (COVID-19): Protecting hospitals from the invisible. Ann Intern Med. 2020; 172(9): 619-620. PMid: 32160299. https://doi.org/10.7326/M20-0751

[7] Grabowski DC, Joynt Maddox KE. Postacute care preparedness for COVID-19: Thinking ahead. JAMA. 2020. PMid: 32211831 https://doi.org/10.1001/jama.2020.4686

[8] Graverholt B, Forsetlund L, Jamtvedt G. Reducing hospital admissions from nursing homes: A systematic review. BMC Health Serv Res. 2014; 14: 36-6963-14-36. PMid: 24456561. https: //doi.org/10.1186/1472-6963-14-36

[9] Levin S, Bonner A, Perry A, et al. COVID-19 in older adults:Transfers between nursing homes and hospitals. Journal of Geriatric Emergency Medicine. 2020; 1(5).

[10] Nyamekye D. Long-term care facilities account for 61 percent of North Carolina's COVID-19 deaths. The Center. May 11, 2020 [Accessed May 19, 2020]. Available from: https://www. thecentersquare.com/north_carolina/1 ong-term-care-facilities-account-for-61-percent-o f-north-carolinas-covid-19-deaths/article_d0ee2672 $-93 c 8-11$ ea-b47e-2338c81ae566.html

[11] Brosseau C. Inspectors find hand-washing neglected in NC nursing homes with COVID-19 outbreaks. News and Observer. Local section. June 15, 2020 [Accessed June 30, 2020]. Available 
from: https://www.newsobserver.com/news/local/articl e243504121.html

[12] Alexander JM, Bonner L. COVID-19 testing in NC nursing homes to be expanded to all residents, staff. News and Observer. June 30, 2020 [Accessed July 3, 2020]. Available from: https://www. news observer.com/article243901297.html

[13] Applegate WB, Ouslander JG. COVID-19 presents high risk to older persons. J Am Geriatr Soc. 2020; 68(4): 681. PMid: 32154911. https://doi.org/10.1111/jgs.16426

[14] North Carolina Department of Health and Human Services. COVID19 North Carolina dashboard. Updated July 2020 [Accessed June 30, 2020]. Available from: https://covid19.ncdhhs.gov/das hboard\#by-congregate-living

[15] Kukka C. States launch rapid response teams to curb COVID-19 outbreaks in nursing homes. National Academy for State Health Policy Web site. Updated April 27, 2020 [Accessed June 4, 2020]. Available from: https: //www.nashp.org/states-launch-rapid-response-teams -to-curb-covid-19-outbreaks-in-nursing-homes/

[16] Cenziper D, Jacobs J, Mulcahy S. Nearly 1 in 10 nursing homes nationwide report coronavirus. The Washington Post. April 20, 2020 [Accessed July 2, 2020]. Available from: https : //www . washingt onpost. com/business/2020/04/20/nearly-one-10-nursi ng-homes-nationwide-report-coronavirus-outbreaks/

[17] Musumeci MB, Chidambaram P. COVID-19 issues and Medicaid policy options for people who need long-term services and support. Kaiser Health News Web site. Updated April 16, 2020 [Accessed May 19, 2020]. Available from: https://www.kff.org/medicaid/issue-brief/covid-1 9-issues-and-medicaid-policy-options-for-people-w ho-need-long-term-services-and-supports/ 